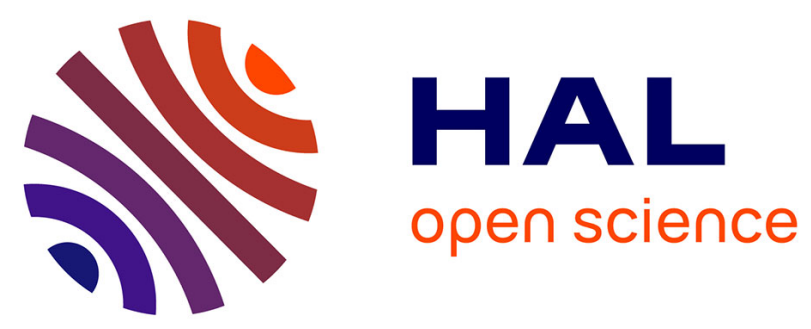

\title{
On a senary quartic form
}

Jianya Liu, Jie Wu, Yongqiang Zhao

\section{To cite this version:}

Jianya Liu, Jie Wu, Yongqiang Zhao. On a senary quartic form. Periodica Mathematica Hungarica, 2020, 80 (2), pp.237-248. 10.1007/s10998-019-00308-y . hal-01865622

\section{HAL Id: hal-01865622 \\ https://hal.science/hal-01865622}

Submitted on 31 Aug 2018

HAL is a multi-disciplinary open access archive for the deposit and dissemination of scientific research documents, whether they are published or not. The documents may come from teaching and research institutions in France or abroad, or from public or private research centers.
L'archive ouverte pluridisciplinaire HAL, est destinée au dépôt et à la diffusion de documents scientifiques de niveau recherche, publiés ou non, émanant des établissements d'enseignement et de recherche français ou étrangers, des laboratoires publics ou privés. 


\title{
ON A SENARY QUARTIC FORM
}

\author{
JIANYA LIU, JIE WU \& YONGQIANG ZHAO
}

ABstract. We count rational points of bounded height on the non-normal senary quartic hypersurface $x^{4}=\left(y_{1}^{2}+\cdots+y_{4}^{2}\right) z^{2}$ in the spirit of Manin's conjecture.

\section{INTRODUCTION}

Recently, we [7] proved Manin's conjecture for singular cubic hypersurfaces

$$
x^{3}=\left(y_{1}^{2}+\cdots+y_{n}^{2}\right) z,
$$

where $n$ is a positive multiple of 4 . In this short note, we show that our method used in [7] also works for higher degree forms like

$$
x^{m}=\left(y_{1}^{2}+\cdots+y_{n}^{2}\right) z^{m-2},
$$

where $n \geqslant 4$ and $m \geqslant 4$. To illustrate, we establish an asymptotic formula for the number of rational points of bounded height on the quartic hypersurface

$$
Q: x^{4}=\left(y_{1}^{2}+y_{2}^{2}+y_{3}^{2}+y_{4}^{2}\right) z^{2},
$$

in the spirit of Manin's conjecture.

It is easy to see that the subvariety $x=z=0$ of $Q$ already contains $\gg B^{4}$ rational points with $|x| \leqslant B,|z| \leqslant B$, and $\left|y_{j}\right| \leqslant B$ with $1 \leqslant j \leqslant 4$, which is predominant and is much larger than the heuristic prediction that is of order $B^{2}$. One therefore counts rational points on the complement subset $U=Q \backslash\{x=z=0\}$. Let $H$ be the height function

$$
H\left(x: y_{1}: \cdots: y_{4}: z\right)=\max \left\{|x|, \sqrt{y_{1}^{2}+\cdots+y_{4}^{2}},|z|\right\}
$$

for $\left(x, y_{1}, \ldots, y_{4}, z\right)=1$. Let $B$ be a large integer, and define

$$
N_{U}(B):=\left|\left\{\left(x: y_{1}: \cdots: y_{4}: z\right) \in U: H\left(x: y_{1}: \cdots: y_{4}: z\right) \leqslant B\right\}\right| .
$$

This counts rational points in $U$ whose height is bounded by $B$, and the aim of this note is obtain an asymptotic formula for it. To this end, we need to understand in advance a similar quantity

$$
N_{U}^{*}(B):=\sum_{\substack{1 \leqslant|x| \leqslant B, 1 \leqslant y_{1}^{2}+\cdots+y_{4}^{2} \leqslant B^{2},|z| \leqslant B \\ x^{4}=\left(y_{1}^{2}+\cdots+y_{4}^{2}\right) z^{2}}} 1 .
$$

One sees, in $N_{U}^{*}(B)$, that the co-prime condition $\left(x, y_{1}, \ldots, y_{4}, z\right)=1$ in $N_{U}(B)$ is relaxed. Our main result is as follows.

Date: August 31, 2018.

2000 Mathematics Subject Classification. 11D45, 11N37.

Key words and phrases. Quartic hypersurface; Manin's conjecture; rational point; asymptotic formula. 
Theorem 1.1. As $B \rightarrow \infty$, we have

$$
\begin{aligned}
& N_{U}(B)=\mathcal{C}_{4} B^{3} \log B\left\{1+O\left(\frac{1}{\sqrt[4]{\log B}}\right)\right\}, \\
& N_{U}^{*}(B)=\mathcal{C}_{4}^{*} B^{3} \log B\left\{1+O\left(\frac{1}{\sqrt[4]{\log B}}\right)\right\}
\end{aligned}
$$

with $\mathcal{C}_{4}:=\frac{192}{5 \zeta(3)} \mathscr{C}_{4}$ and $\mathcal{C}_{4}^{*}:=\frac{192}{5} \mathscr{C}_{4}$, where $\mathscr{C}_{4}$ is defined as in $(2.6)$ below, and $\zeta$ is the Riemann zeta-function.

We note that the exponent of $B$ in the main terms of the above theorem is 3 instead of 2 as predicted by the usual heuristic. This phenomenon may be explained by the fact that the hypersurface $Q$ is not normal.

It is easy to check that $Q$ has an obvious quadric bundle structure given by

$$
Q_{[a: b]}:\left\{\begin{array}{l}
b^{2} x^{2}=a^{2}\left(y_{1}^{2}+y_{2}^{2}+y_{3}^{2}+y_{4}^{2}\right), \\
a x-b y=0
\end{array}\right.
$$

and $\left\{Q_{[a: b]}\right\}$ covers $Q$ as long as $[a: b]$ goes thorough $\mathbb{P}^{1}(\mathbb{Q})$. From this, it is possible to interpret Theorem 1.1 in the framework of the generalized Manin's conjecture by Batyrev and Tschinkel [1], as was done in the work of de la Bretèche, Browning, and Salberger [3]. However, we will not pursue such an explanation here. The only sole purpose of this short note is to show that our method used in [7] also works for higher degree forms $Q$.

Finally, we remark that using the method in our joint paper [4] with de la Bretèche, one can get power-saving error terms in Theorem 1.1, which we will not pursue here.

\section{Outline of the Proof of Theorem 1.1}

Denote by $r_{4}(d)$ the number representations of a positive integer $d$ as the sum of four squares : $d=y_{1}^{2}+\cdots+y_{4}^{2}$ with $\left(y_{1}, \ldots, y_{4}\right) \in \mathbb{Z}^{4}$. It is well-known (cf. [5, (3.9)]) that

$$
r_{4}(d)=8 r_{4}^{*}(d) \quad \text { with } \quad r_{4}^{*}(d):=\sum_{\ell \mid d, \ell \neq 0(\bmod 4)} \ell .
$$

Let $\mathbb{1}_{\square}(n)$ be the characteristic function of squares. In view of the above, we can write

$$
N_{U}^{*}(B)=32\left\{\sum_{n \leqslant B} \sum_{\substack{d \mid n^{4} \\ d \leqslant B^{2}}} r_{4}^{*}(d) \mathbb{1}_{\square}\left(\frac{n^{4}}{d}\right)-\sum_{n \leqslant B} \sum_{\substack{d \mid n^{4} \\ d<n^{4} / B^{2}}} r_{4}^{*}(d) \mathbb{1}_{\square}\left(\frac{n^{4}}{d}\right)\right\} .
$$

Hence to prove (1.5) in Theorem 1.1, it is sufficient to establish asymptotic formulae for the following two quantities

$$
S(x, y):=\sum_{n \leqslant x} \sum_{\substack{d \mid n^{4} \\ d \leqslant y}} r_{4}^{*}(d) \mathbb{1}_{\square}\left(\frac{n^{4}}{d}\right), \quad T(B):=\sum_{n \leqslant B} \sum_{\substack{d \mid n^{4} \\ d<n^{4} / B^{2}}} r_{4}^{*}(d) \mathbb{1}_{\square}\left(\frac{n^{4}}{d}\right) .
$$

For $S(x, y)$, our result is as follows. 
Theorem 2.1. Let $\varepsilon>0$ be arbitrary. We have

$$
S(x, y)=x y\left(4 P(\psi)+\frac{3}{2} P^{\prime}(\psi)\right)+O_{\varepsilon}\left(x^{\frac{5}{4}} y^{\frac{7}{8}}+x^{\frac{1}{2}+\varepsilon} y^{\frac{9}{8}}\right)
$$

uniformly for $x^{3} \geqslant y \geqslant x \geqslant 10$, where $\psi:=\log x-\frac{1}{4} \log y$ and $P(t)$ is a quadratic polynomial, defined as in (4.18) below. In particular, for any fixed $\eta \in(0,1]$ we have

$$
S(x, y)=4 \mathscr{C}_{4} x y\left(\log x-\frac{1}{4} \log y\right)\left\{1+O\left(\frac{1}{(\log x)^{\eta}}\right)\right\}
$$

uniformly for $x \geqslant 10$ and $x^{2}(\log x)^{-8(1-\eta)} \leqslant y \leqslant x^{3}$, where

$$
\mathscr{C}_{4}:=\frac{23}{150} \zeta(5) \prod_{p}\left(1+\frac{1}{p}+\frac{2}{p^{2}}+\frac{2}{p^{3}}+\frac{1}{p^{4}}+\frac{1}{p^{5}}\right)\left(1-\frac{1}{p}\right)
$$

is the leading coefficient of $P(t)$.

Now we turn to analyze $T(B)$ which is more difficult, since the range of its second summation depends on the variable $n$ of the first summation. Thus Theorem 2.1 does not apply to $T(B)$ directly. In $\S 5$ we show that Theorem 2.1 together with delicate analysis is sufficient to establish the following result.

Theorem 2.2. As $B \rightarrow \infty$, we have

$$
T(B)=\frac{2}{5} \mathscr{C}_{4} B^{3} \log B\left\{1+O\left(\frac{1}{\sqrt[4]{\log B}}\right)\right\}
$$

where $\mathscr{C}_{4}$ is as in (2.6) above.

As in $[2,7]$, we shall firstly establish an asymptotic formula for the quantity

$$
M(X, Y):=\int_{1}^{Y} \int_{1}^{X} S(x, y) \mathrm{d} x \mathrm{~d} y
$$

by applying the method of complex integration. Then we derive the asymptotic formula (2.4) for $S(x, y)$ in Theorem 2.1 by the operator $\mathscr{D}$ defined below. Let $\mathscr{E}_{k}$ be the set of all functions of $k$ variables. Define the operator $\mathscr{D}: \mathscr{E}_{2} \rightarrow \mathscr{E}_{4}$ by

$$
(\mathscr{D} f)(X, H ; Y, J):=f(H, J)-f(H, Y)-f(X, J)+f(X, Y) .
$$

The next lemma summarises all properties of $\mathscr{D}$ needed later.

Lemma 2.1. (i) Let $f \in \mathscr{E}_{2}$ be a function of class $C^{3}$. Then we have

$$
(\mathscr{D} f)(X, H ; Y, J)=(J-Y)(H-X)\left\{\frac{\partial^{2} f}{\partial x \partial y}(X, Y)+O(R(X, H ; Y, J))\right\}
$$

for $X \leqslant H$ and $Y \leqslant J$, where

$$
R(X, H ; Y, J):=(H-X) \max _{\substack{X \leqslant x \leqslant H \\ Y \leqslant y \leqslant J}}\left|\frac{\partial^{3} f}{\partial x^{2} \partial y}(x, y)\right|+(J-Y) \max _{\substack{X \leqslant x \leqslant H \\ Y \leqslant y \leqslant J}}\left|\frac{\partial^{3} f}{\partial x \partial y^{2}}(x, y)\right| .
$$

(ii) Let $S(x, y)$ and $M(X, Y)$ be defined as in (2.3) and (2.8). Then

$$
(\mathscr{D} M)(X-H, X ; Y-J, Y) \leqslant H J S(X, Y) \leqslant(\mathscr{D} M)(X, X+H ; Y, Y+J)
$$

for $H \leqslant X$ and $J \leqslant Y$. 
The next elementary estimate ([2, Lemma 6(i)] or [7, Lemma 4.3]) will also be used several times in the paper.

Lemma 2.2. Let $1 \leqslant H \leqslant X$ and $|\sigma| \leqslant 10$. Then for any $\beta \in[0,1]$, we have

$$
\left|(X+H)^{s}-X^{s}\right| \ll X^{\sigma}((|\tau|+1) H / X)^{\beta},
$$

where the implied constant is absolute.

\section{Dirichlet Series associated with $S(x, y)$}

In view of the definition of $S(x, y)$ in $(2.3)$, we define the double Dirichlet series

$$
\mathcal{F}(s, w):=\sum_{n \geqslant 1} n^{-s} \sum_{d \mid n^{4}} d^{-w} r_{4}^{*}(d) \mathbb{1}_{\square}\left(\frac{n^{4}}{d}\right)
$$

for $\Re e s>5$ and $\Re e w>0$. The next lemma states that the function $\mathcal{F}(s, w)$ enjoys a nice factorization formula.

Lemma 3.1. For $\min _{0 \leqslant j \leqslant 2} \Re e(s+2 j w-2 j)>1$, we have

$$
\mathcal{F}(s, w)=\prod_{0 \leqslant j \leqslant 2} \zeta(s+2 j w-2 j) \mathcal{G}(s, w),
$$

where $\mathcal{G}(s, w)$ is an Euler product, given by (3.8), (3.10) and (3.11) below. Further, for any $\varepsilon>0$ and for $\min _{0 \leqslant j \leqslant 2} \Re e(s+2 j w-2 j) \geqslant \frac{1}{2}+\varepsilon, \mathcal{G}(s, w)$ converges absolutely and

$$
\mathcal{G}(s, w) \ll_{\varepsilon} 1 .
$$

Proof. Since the functions $r_{4}^{*}(d)$ and $n^{-s} \sum_{d \mid n^{4}} d^{-w} r_{4}^{*}(d) \mathbb{1}_{\square}\left(n^{4} / d\right)$ are multiplicative, for $\Re e s>5$ and $\Re e w>0$ we can write the Euler product

$$
\mathcal{F}(s, w)=\prod_{p} \sum_{\nu \geqslant 0} p^{-\nu s} \sum_{0 \leqslant \mu \leqslant 2 \nu} p^{-2 \mu w} r_{4}^{*}\left(p^{2 \mu}\right)=\prod_{p} \mathcal{F}_{p}(s, w) .
$$

In the above computations, speacial attention should be paid to the effect of the function $\mathbb{1}_{\square}$. The next is to simplify each $\mathcal{F}_{p}(s, w)$. To this end, we recall $(2.1)$ so that

$$
r_{4}^{*}\left(p^{\mu}\right)=\frac{1-p^{\mu+1}}{1-p} \quad(p>2), \quad r_{4}^{*}\left(2^{\mu}\right)=3
$$

for all integers $\mu \geqslant 1$. On the other hand, a simple formal calculation shows

$$
\begin{aligned}
\sum_{\nu \geqslant 0} x^{\nu} \sum_{0 \leqslant \mu \leqslant 2 \nu} y^{2 \mu} \frac{1-z^{2 \mu+1}}{1-z} & =\frac{1}{1-z} \sum_{\nu \geqslant 0} x^{\nu}\left(\frac{1-y^{4 \nu+2}}{1-y^{2}}-z \frac{1-(y z)^{4 \nu+2}}{1-y^{2} z^{2}}\right) \\
& =\frac{1+x y^{2}\left(1+z+z^{2}\right)+x y^{4}\left(z+z^{2}+z^{3}\right)+x^{2} y^{6} z^{3}}{(1-x)\left(1-x y^{4}\right)\left(1-x y^{4} z^{4}\right)}
\end{aligned}
$$

and

$$
\begin{aligned}
1+\sum_{\nu \geqslant 1} x^{\nu}\left(1+a \sum_{1 \leqslant \mu \leqslant 2 \nu} y^{2 \mu}\right) & =1+\sum_{\nu \geqslant 1} x^{\nu}\left(1+a \frac{y^{2}-y^{4 \nu+2}}{1-y^{2}}\right) \\
& =\frac{1+a x y^{2}+(a-1) x y^{4}}{(1-x)\left(1-x y^{4}\right)} .
\end{aligned}
$$


When $p>2$, in view of (3.4), we can apply (3.5) with $(x, y, z)=\left(p^{-s}, p^{-w}, p\right)$ to write

$$
\mathcal{F}_{p}(s, w)=\prod_{0 \leqslant j \leqslant 2}\left(1-p^{-(s+2 j w-2 j)}\right)^{-1} \mathcal{G}_{p}(s, w),
$$

where

$$
\begin{aligned}
& \mathcal{G}_{p}(s, w) \\
& :=\left(1+\frac{p^{2}+p+1}{p^{s+2 w}}+\frac{p^{3}+p^{2}+p}{p^{s+4 w}}+\frac{p^{3}}{p^{2 s+6 w}}\right)\left(1-\frac{p^{2}}{p^{s+2 w}}\right)\left(1-\frac{1}{p^{s+4 w}}\right)^{-1} .
\end{aligned}
$$

While for $p=2$, the formula (3.6) with $(x, y, z, a)=\left(2^{-s}, 2^{-w}, 2,3\right)$ gives

$$
\mathcal{F}_{2}(s, w)=\prod_{0 \leqslant j \leqslant 2}\left(1-2^{-(s+2 j w-2 j)}\right)^{-1} \mathcal{G}_{2}(s, w),
$$

where

$$
\mathcal{G}_{2}(s, w):=\frac{1+3 \cdot 2^{-s-2 w}+2^{-s-4 w+1}}{1-2^{-s-4 w}} \prod_{1 \leqslant j \leqslant 2}\left(1-2^{-(s+2 j w-2 j)}\right) .
$$

Combining (3.7)-(3.10), we get (3.2) with

$$
\mathcal{G}(s, w):=\prod_{p} \mathcal{G}_{p}(s, w) \quad(\Re e s>5, \Re e w>0) .
$$

It is easy to verify that for $\min _{0 \leqslant j \leqslant 2}(\sigma+2 j u-2 j) \geqslant \frac{1}{2}+\varepsilon$, we have $\left|\mathcal{G}_{p}(s, w)\right|=$ $1+O\left(p^{-1-\varepsilon}\right)$. This shows that under the same condition, the Euler product $\mathcal{G}(s, w)$ converges absolutely and (3.3) holds. By analytic continuation, (3.2) is also true in the same domain. This completes the proof.

\section{Proof of Theorem 2.1}

In the sequel, we suppose

$$
10 \leqslant X \leqslant Y \leqslant X^{3}, \quad(X Y)^{3} \leqslant 4 T \leqslant U \leqslant X^{12}, \quad H \leqslant X, \quad J \leqslant Y,
$$

and for brevity we fix the following notation:

$$
s:=\sigma+\mathrm{i} \tau, \quad w:=u+\mathrm{i} v, \quad \mathcal{L}:=\log X, \quad \kappa:=1+\mathcal{L}^{-1}, \quad \lambda:=1+4 \mathcal{L}^{-1} .
$$

The following proposition is an immediate consequence of Lemmas 4.2-4.5 below.

Proposition 4.1. Under the previous notation, we have

$$
M(X, Y)=X^{2} Y^{2} P\left(\log X-\frac{1}{4} \log Y\right)+R_{0}(X, Y)+R_{1}(X, Y)+R_{2}(X, Y)+O(1)
$$

uniformly for $(X, Y, T, U, H, J)$ satisfying (4.1), where $R_{0}, R_{1}, R_{2}$ and $P(t)$ are defined as in (4.8), (4.13), (4.16) and (4.18) below, respectively.

The proof is divided into several subsections. 
4.1. Application of Perron's formula. The first step is to apply Perron's formula twice to transform $M(X, Y)$ into a form that is ready for future treatment.

Lemma 4.2. Under the previous notation, we have

$$
M(X, Y)=M(X, Y ; T, U)+O(1)
$$

uniformly for $(X, Y, T, U)$ satisfying (4.1), where the implied constant is absolute and

$$
M(X, Y ; T, U):=\frac{1}{(2 \pi \mathrm{i})^{2}} \int_{\kappa-\mathrm{i} T}^{\kappa+\mathrm{i} T}\left(\int_{\lambda-\mathrm{i} U}^{\lambda+\mathrm{i} U} \frac{\mathcal{F}(s, w) Y^{w+1}}{w(w+1)} \mathrm{d} w\right) \frac{X^{s+1}}{s(s+1)} \mathrm{d} s .
$$

The proof is the same as that of [7, Lemma 6.2].

4.2. Application of Cauchy's theorem. In this subsection, we shall apply Cauchy's theorem to evaluate the integral over $w$ in $M(X, Y ; T, U)$. We write

$$
w_{j}=w_{j}(s):=(2 j+1-s) /(2 j) \quad(1 \leqslant j \leqslant 2)
$$

and

$$
\mathcal{F}_{1}^{*}(s):=\zeta(s) \zeta(2-s) \mathcal{G}\left(s, w_{1}(s)\right), \quad \mathcal{F}_{2}^{*}(s):=\zeta(s) \zeta\left(\frac{s+1}{2}\right) \mathcal{G}\left(s, w_{2}(s)\right) .
$$

Lemma 4.3. Under the previous notation, for any $\varepsilon>0$ we have

$$
M(X, Y ; T, U)=I_{1}+I_{2}+R_{0}(X, Y)+O_{\varepsilon}(1)
$$

uniformly for $(X, Y, T, U)$ satisfying (4.1), where

$$
\begin{aligned}
& I_{1}:=\frac{4}{2 \pi \mathrm{i}} \int_{\kappa-\mathrm{i} T}^{\kappa+\mathrm{i} T} \frac{\mathcal{F}_{1}^{*}(s) X^{s+1} Y^{(5-s) / 2}}{(3-s)(5-s) s(s+1)} \mathrm{d} s, \\
& I_{2}:=\frac{16}{2 \pi \mathrm{i}} \int_{\kappa-\mathrm{i} T}^{\kappa+\mathrm{i} T} \frac{\mathcal{F}_{2}^{*}(s) X^{s+1} Y^{(9-s) / 4}}{(5-s)(9-s) s(s+1)} \mathrm{d} s,
\end{aligned}
$$

and

$$
R_{0}(X, Y):=\frac{1}{(2 \pi \mathrm{i})^{2}} \int_{\kappa-\mathrm{i} T}^{\kappa+\mathrm{i} T}\left(\int_{\frac{11}{12}+\varepsilon-\mathrm{i} U}^{\frac{11}{12}+\varepsilon+\mathrm{i} U} \frac{\mathcal{F}(s, w) Y^{w+1}}{w(w+1)} \mathrm{d} w\right) \frac{X^{s+1}}{s(s+1)} \mathrm{d} s .
$$

Furthermore we have

$$
\left.\begin{array}{l}
\left(\mathscr{D} R_{0}\right)(X, X+H ; Y, Y+J) \\
\left(\mathscr{D} R_{0}\right)(X-H, X ; Y-J, Y)
\end{array}\right\} \ll_{\varepsilon} X^{\frac{7}{6}+\varepsilon} Y^{\frac{11}{12}+\varepsilon} H^{\frac{5}{6}} J+X^{1+\varepsilon} Y^{\frac{13}{12}+\varepsilon} H J^{\frac{5}{6}}
$$

uniformly for $(X, Y, T, U, H, J)$ satisfying (4.1).

Proof. We want to calculate the integral

$$
\frac{1}{2 \pi \mathrm{i}} \int_{\lambda-\mathrm{i} U}^{\lambda+\mathrm{i} U} \frac{\mathcal{F}(s, w) Y^{w+1}}{w(w+1)} \mathrm{d} w
$$

for any individual $s=\sigma+\mathrm{i} \tau$ with $\sigma=\kappa$ and $|\tau| \leqslant T$. We move the line of integration $\Re e w=\lambda$ to $\Re e w=\frac{3}{4}+\varepsilon$. By Lemma 3.1, for $\sigma=\kappa$ and $|\tau| \leqslant T$, the points $w_{j}(s)(j=1,2)$, given by $(4.5)$, are the simple poles of the integrand in the rectangle $\frac{3}{4}+\varepsilon \leqslant u \leqslant \lambda$ and $|v| \leqslant U$. The residues of $\frac{\mathcal{F}(s, w)}{w(w+1)} Y^{w+1}$ at the poles $w_{j}(s)$ are

$$
\frac{4 \mathcal{F}_{1}^{*}(s) Y^{(5-s) / 2}}{(3-s)(5-s)}, \quad \frac{16 \mathcal{F}_{2}^{*}(s) Y^{(9-s) / 4}}{(5-s)(9-s)},
$$


respectively, where $\mathcal{F}_{j}^{*}(s)(j=1,2)$ are defined as in (4.6).

It is well-known that (cf. e.g. [8, page 146, Theorem II.3.7])

$$
\zeta(s) \ll|\tau|^{\max \{(1-\sigma) / 3,0\}} \log |\tau| \quad\left(\sigma \geqslant \frac{1}{2},|\tau| \geqslant 2\right)
$$

where $c>0$ is a constant. When $\sigma=\kappa$ and $\frac{11}{12}+\varepsilon \leqslant u \leqslant \lambda$, it is easily checked that

$$
\min _{0 \leqslant j \leqslant 2}(\sigma+2 j u-2 j) \geqslant 1+4\left(\frac{11}{12}+\varepsilon-1\right)=\frac{3}{4}+3 \varepsilon>\frac{1}{2}+\varepsilon .
$$

It follows from (4.11) and (3.3) that $\mathcal{F}(s, w) \ll_{\varepsilon} U^{2(1-u)} \mathcal{L}^{4}$ for $\sigma=\kappa,|\tau| \leqslant T, \frac{11}{12}+\varepsilon \leqslant$ $u \leqslant \lambda$ and $v= \pm U$. This implies that

$$
\int_{\frac{11}{12}+\varepsilon \pm \mathrm{i} U}^{\lambda \pm \mathrm{i} U} \frac{\mathcal{F}(s, w) Y^{w+1}}{w(w+1)} \mathrm{d} w \ll_{\varepsilon} Y \mathcal{L}^{4} \int_{\frac{11}{12}}^{\lambda}\left(\frac{Y}{U^{2}}\right)^{u} \mathrm{~d} u \ll_{\varepsilon} \frac{Y^{\frac{23}{12}} \mathcal{L}^{4}}{U^{\frac{11}{6}}} \ll_{\varepsilon} 1 .
$$

Cauchy's theorem then gives

$$
\begin{aligned}
\frac{1}{2 \pi \mathrm{i}} \int_{\lambda-\mathrm{i} U}^{\lambda+\mathrm{i} U} \frac{\mathcal{F}(s, w) Y^{w+1}}{w(w+1)} \mathrm{d} w= & \frac{4 \mathcal{F}_{1}^{*}(s) Y^{(5-s) / 2}}{(3-s)(5-s)}+\frac{16 \mathcal{F}_{2}^{*}(s) Y^{(9-s) / 4}}{(5-s)(9-s)} \\
& +\frac{1}{2 \pi \mathrm{i}} \int_{\frac{11}{12}+\varepsilon-\mathrm{i} U}^{\frac{11}{12}+\varepsilon+\mathrm{i} U} \frac{\mathcal{F}(s, w) Y^{w+1}}{w(w+1)} \mathrm{d} w+O_{\varepsilon}(1) .
\end{aligned}
$$

Inserting the last formula into (4.4), we obtain (4.7).

Finally we prove (4.9). For $\sigma=\kappa,|\tau| \leqslant T, u=\frac{11}{12}+\varepsilon$ and $|v| \leqslant U$, we apply (4.11) and (3.3) as before, to get

$$
\mathcal{F}(s, w) \ll(|\tau|+|v|+1)^{\frac{1}{6}} \mathcal{L}^{4} \ll\left\{(|\tau|+1)^{\frac{1}{6}}+(|v|+1)^{\frac{1}{6}}\right\} \mathcal{L}^{4} .
$$

Also, for $\sigma, \tau, u, v$ as above, we have

$$
\begin{aligned}
r_{s, w}(X, H ; Y, J) & :=\left((X+H)^{s+1}-X^{s+1}\right)\left((Y+J)^{w+1}-Y^{w+1}\right) \\
& \left.\ll X^{2}((|\tau|+1) H / X)\right)^{\frac{5}{6}-\varepsilon} Y^{\frac{23}{12}+\varepsilon}((|v|+1) J / Y)^{1-\varepsilon} \\
& \ll X^{\frac{7}{6}+\varepsilon} Y^{\frac{11}{12}+\varepsilon} H^{\frac{5}{6}} J(|\tau|+1)^{\frac{5}{6}-\varepsilon}(|v|+1)^{1-\varepsilon}
\end{aligned}
$$

by (2.10) of Lemma 2.2 with $\beta=\frac{5}{6}-\varepsilon$ and with $\beta=1-\varepsilon$. Similarly,

$$
\begin{aligned}
r_{s, w}(X, H ; Y, J) & =\left((X+H)^{s+1}-X^{s+1}\right)\left((Y+J)^{w+1}-Y^{w+1}\right) \\
& \left.\ll X^{2}((|\tau|+1) H / X)\right)^{1-\varepsilon} Y^{\frac{23}{12}+\varepsilon}((|v|+1) J / Y)^{\frac{5}{6}-\varepsilon} \\
& \ll X^{1+\varepsilon} Y^{\frac{13}{12}+\varepsilon} H J^{\frac{5}{6}}(|\tau|+1)^{1-\varepsilon}(|v|+1)^{\frac{5}{6}-\varepsilon}
\end{aligned}
$$

by Lemma 2.2 with $\beta=1-\varepsilon$ and with $\beta=\frac{5}{6}-\varepsilon$. These and Lemma 2.1(i) imply

$$
\begin{aligned}
\left(\mathscr{D} R_{0}\right)(X, X+H ; Y, Y+J) & =\int_{\kappa-\mathrm{i} T}^{\kappa+\mathrm{i} T} \int_{\frac{11}{12}+\varepsilon-\mathrm{i} U}^{\frac{11}{12}+\varepsilon+\mathrm{i} U} \frac{\mathcal{F}(s, w)}{(2 \pi \mathrm{i})^{2}} \frac{r_{s, w}(X, H ; Y, J)}{s(s+1) w(w+1)} \mathrm{d} w \mathrm{~d} s \\
& \ll_{\varepsilon} X^{\frac{7}{6}+\varepsilon} Y^{\frac{11}{12}+\varepsilon} H^{\frac{5}{6}} J+X^{1+\varepsilon} Y^{\frac{13}{12}+\varepsilon} H J^{\frac{5}{6}} .
\end{aligned}
$$

This completes the proof. 


\subsection{Evaluation of $I_{1}$.}

Lemma 4.4. Under the previous notation, we have

$$
I_{1}=R_{1}(X, Y)+O(1)
$$

uniformly for $(X, Y, T)$ satisfying (4.1), where

$$
R_{1}(X, Y):=\frac{4}{2 \pi \mathrm{i}} \int_{\frac{5}{4}-\mathrm{i} T}^{\frac{5}{4}+\mathrm{i} T} \frac{\mathcal{F}_{1}^{*}(s) X^{s+1} Y^{(5-s) / 2}}{(3-s)(5-s) s(s+1)} \mathrm{d} s .
$$

Further we have

$$
\left.\begin{array}{l}
\left(\mathscr{D} R_{1}\right)(X, X+H ; Y, Y+J) \\
\left(\mathscr{D} R_{1}\right)(X-H, X ; Y-J, Y)
\end{array}\right\} \ll X^{\frac{5}{4}} Y^{\frac{7}{8}} H J
$$

uniformly for $(X, Y, T, H, J)$ satisfying (4.1).

Proof. We shall prove (4.12) by moving the contour $\Re e s=\kappa$ to $\Re e s=\frac{5}{4}$. When $\kappa \leqslant \sigma \leqslant \frac{5}{4}$, it is easy to check that

$$
\min _{0 \leqslant j \leqslant 2}\left(\sigma+2 j w_{1}(\sigma)-2 j\right)=\min _{0 \leqslant j \leqslant 2}(j+(1-j) \sigma) \geqslant \frac{3}{4} .
$$

By Lemma 3.1 the integrand is holomorphic in the rectangle $\kappa \leqslant \sigma \leqslant \frac{5}{4}$ and $|\tau| \leqslant T$; and we can apply (4.11) and (3.3) to get $\mathcal{F}_{1}^{*}(s) \ll T^{(\sigma-1) / 3} \mathcal{L}^{2}$ in this rectangle, which implies that

$$
\begin{aligned}
\int_{\kappa \pm \mathrm{i} T}^{\frac{5}{4} \pm \mathrm{i} T} \frac{\mathcal{F}_{1}^{*}(s) X^{s+1} Y^{(5-s) / 2}}{(3-s)(5-s) s(s+1)} \mathrm{d} s & \ll \frac{X^{2} Y^{2} \mathcal{L}^{2}}{T^{4}} \int_{\kappa}^{\frac{5}{4}}\left(\frac{X T^{1 / 3}}{Y^{1 / 2}}\right)^{\sigma-1} \mathrm{~d} \sigma \\
& \ll \frac{X^{2} Y^{2} \mathcal{L}^{2}}{T^{4}}+\frac{X^{\frac{9}{4}} Y^{\frac{15}{8}} \mathcal{L}^{2}}{T^{\frac{47}{12}}} \ll 1 .
\end{aligned}
$$

This proves (4.12).

To establish (4.14), we note that $\mathcal{F}_{1}^{*}(s) \ll(|\tau|+1)^{\frac{1}{4}}$ for $\sigma=\frac{5}{4}$ and $|\tau| \leqslant T$. By (2.10) of Lemma 2.2 with $\beta=1$,

$$
\begin{aligned}
r_{s, w_{1}(s)}(X, H ; Y, J) & :=\left((X+H)^{s+1}-X^{s+1}\right)\left((Y+J)^{(5-s) / 2}-Y^{(5-s) / 2}\right) \\
& \ll X^{\frac{5}{4}} Y^{\frac{7}{8}} H J(|\tau|+1)^{2} .
\end{aligned}
$$

Combining these with Lemma 2.1(ii), we deduce that

$$
\begin{aligned}
\left(\mathscr{D} R_{1}\right)(X, X+H ; Y, Y+J) & =\frac{4}{2 \pi \mathrm{i}} \int_{\frac{5}{4}-\mathrm{i} T}^{\frac{5}{4}+\mathrm{i} T} \frac{\mathcal{F}_{1}^{*}(s) r_{s, w_{1}(s)}(X, H ; Y, J)}{(3-s)(5-s) s(s+1)} \mathrm{d} s \\
& \ll X^{\frac{5}{4}} Y^{\frac{7}{8}} H J
\end{aligned}
$$

from which the desired result follows. 


\subsection{Evaluation of $I_{2}$.}

Lemma 4.5. Under the previous notation, for any $\varepsilon>0$ we have

$$
I_{2}=X^{2} Y^{2} P\left(\log X-\frac{1}{4} \log Y\right)+R_{2}(X, Y)+O_{\varepsilon}(1)
$$

uniformly for $(X, Y, T)$ satisfying $(4.1)$, where $P(t)$ is defined as in (4.18) below and

$$
R_{2}(X, Y):=\frac{16}{2 \pi \mathrm{i}} \int_{\frac{1}{2}+\varepsilon-\mathrm{i} T}^{\frac{1}{2}+\varepsilon+\mathrm{i} T} \frac{\mathcal{F}_{2}^{*}(s) X^{s+1} Y^{(9-s) / 4}}{(5-s)(9-s) s(s+1)} \mathrm{d} s .
$$

Further we have

$$
\left.\begin{array}{l}
\left(\mathscr{D} R_{2}\right)(X, X+H ; Y, Y+J) \\
\left(\mathscr{D} R_{2}\right)(X-H, X ; Y-J, Y)
\end{array}\right\} \ll_{\varepsilon} X^{\frac{1}{2}+\varepsilon} Y^{\frac{9}{8}} H J
$$

uniformly for $(X, Y, T, H, J)$ satisfying (4.1).

Proof. We move the line of integration $\Re e s=\kappa$ to $\Re e s=\frac{1}{2}+\varepsilon$. Obviously $s=1$ is the unique pole of order 2 of the integrand in the rectangle $\frac{1}{2}+\varepsilon \leqslant \sigma \leqslant \kappa$ and $|\tau| \leqslant T$, and the residue is $X^{2} Y^{2} P\left(\log X-\frac{1}{4} \log Y\right)$ with

$$
P(t):=\left.\left(\frac{16(s-1)^{2} \mathcal{F}_{2}^{*}(s) \mathrm{e}^{t(s-1)}}{(5-s)(9-s) s(s+1)}\right)^{\prime}\right|_{s=1} .
$$

Here $P(t)$ is a linear polynomial with the leading coefficient $\mathscr{C}_{4}$ given by (2.6) above.

When $\frac{1}{2}+\varepsilon \leqslant \sigma \leqslant \kappa$, we check that

$$
\min _{0 \leqslant j \leqslant 2}\left(\sigma+2 j w_{2}(\sigma)-2 j\right)=\frac{1}{2} \min _{0 \leqslant j \leqslant 2}(j+(2-j) \sigma) \geqslant \frac{1}{2}+\varepsilon .
$$

Hence when $\frac{1}{2}+\varepsilon \leqslant \sigma \leqslant \kappa$ and $|\tau| \leqslant T$, (4.11) and (3.3) yields $\mathcal{F}_{2}^{*}(s) \ll T^{(1-\sigma) / 2} \mathcal{L}^{3}$. It follows that

$$
\begin{aligned}
\int_{\frac{1}{2}+\varepsilon \pm \mathrm{i} T}^{\kappa \pm \mathrm{i} T} \frac{\mathcal{F}_{2}^{*}(s) X^{s+1} Y^{(9-s) / 4}}{(5-s)(9-s) s(s+1)} \mathrm{d} s & \ll \frac{X^{2} Y^{2} \mathcal{L}^{3}}{T^{4}} \int_{\frac{1}{2}}^{\kappa}\left(\frac{Y T^{2}}{X^{4}}\right)^{(1-\sigma) / 4} \mathrm{~d} s \\
& \ll \frac{X^{2} Y^{2} \mathcal{L}^{3}}{T^{4}}+\frac{X^{\frac{3}{2}} Y^{\frac{9}{4}} \mathcal{L}^{3}}{T^{\frac{7}{2}}} \ll 1 .
\end{aligned}
$$

These establish (4.15). To prove (4.17), we note that for $\sigma=\frac{1}{2}+\varepsilon$ and $|\tau| \leqslant T$, we have $\mathcal{F}_{2}^{*}(s) \ll_{\varepsilon}(|\tau|+1)^{1 / 3}$ thanks to $(4.11)$ and $(3.3)$, and

$$
\begin{aligned}
r_{s, w_{2}(s)}(X, H ; Y, J) & :=\left((X+H)^{s+1}-X^{s+1}\right)\left((Y+J)^{(9-s) / 4}-Y^{(9-s) / 4}\right) \\
& \ll_{\varepsilon} X^{\frac{1}{2}+\varepsilon} Y^{\frac{9}{8}} H J(|\tau|+1)^{2}
\end{aligned}
$$

by Lemma 2.2 with $\beta=1$. Combining these with Lemma 2.1(i), we deduce that

$$
\begin{aligned}
\left(\mathscr{D} R_{2}\right)(X, X+H ; Y, Y+J) & =\frac{16}{2 \pi \mathrm{i}} \int_{\frac{1}{2}+\varepsilon-\mathrm{i} T}^{\frac{1}{2}+\varepsilon+\mathrm{i} T} \frac{\mathcal{F}_{2}^{*}(s) r_{s, w_{2}(s)}(X, H ; Y, J)}{(5-s)(9-s) s(s+1)} \mathrm{d} s \\
& \ll_{\varepsilon} X^{\frac{1}{2}+\varepsilon} Y^{\frac{9}{8}} H J .
\end{aligned}
$$

This proves the lemma. 
4.5. Completion of proof of Theorem 2.1. Denote by $\mathcal{M}(X, Y)$ the main term in the asymptotic formula of $M(x, y)$ in Proposition 4.1, that is $\mathcal{M}(X, Y):=X^{2} Y^{2} P(\psi)$ and $\psi:=\log \left(X / Y^{1 / 4}\right)$. Then Lemma 2.1(i) gives

$$
(\mathscr{D \mathcal { M }})(X, X+H ; Y, Y+J)=\left\{X Y\left(4 P(\psi)+\frac{3}{2} P^{\prime}(\psi)\right)+O\left(X J \mathcal{L}^{2}+Y H \mathcal{L}^{2}\right)\right\} H J .
$$

Since $\mathscr{D}$ is a linear operator, this together with Proposition 4.1 implies that

$$
(\mathscr{D} M)(X, X+H ; Y, Y+J)=\left\{X Y\left(4 P(\psi)+\frac{3}{2} P^{\prime}(\psi)\right)+O_{\varepsilon}(\mathcal{R})\right\} H J
$$

with

$$
\mathcal{R}:=X^{\frac{7}{6}+\varepsilon} Y^{\frac{11}{12}} H^{-\frac{1}{6}}+X^{1+\varepsilon} Y^{\frac{13}{12}} J^{-\frac{1}{6}}+X^{\frac{5}{4}} Y^{\frac{7}{8}}+X^{\frac{1}{2}+\varepsilon} Y^{\frac{9}{8}}+X J \mathcal{L}^{2}+Y H \mathcal{L}^{2} .
$$

The same formula also holds for $(\mathscr{D} M)(X-H, X ; Y-J, Y)$. Now Lemma 2.1(ii) with $H=X Y^{-\frac{1}{14}}$ and $J=Y^{\frac{13}{14}}$ allows us to deduce

$$
S(X, Y)=X Y\left(4 P(\psi)+\frac{3}{2} P^{\prime}(\psi)\right)+O_{\varepsilon}\left(X^{\frac{5}{4}} Y^{\frac{7}{8}}+X^{\frac{1}{2}+\varepsilon} Y^{\frac{9}{8}}\right),
$$

where we have used the following facts

$$
\begin{gathered}
\left(X^{\frac{5}{4}} Y^{\frac{7}{8}}\right)^{\frac{5-24 \varepsilon}{6}}\left(X^{\frac{1}{2}+\varepsilon} Y^{\frac{9}{8}}\right)^{\frac{1+24 \varepsilon}{6}}=X^{\frac{27}{24}-\frac{4(17-24 \varepsilon) \varepsilon}{24}} Y^{\frac{11}{12}+\varepsilon} \geqslant X^{1+\varepsilon} Y^{\frac{11}{12}+\varepsilon}, \\
\left(X^{\frac{5}{4}} Y^{\frac{7}{8}}\right)^{\frac{11}{14}}\left(X^{\frac{1}{2}+\varepsilon} Y^{\frac{9}{8}}\right)^{\frac{3}{14}}=X^{\frac{61+12 \varepsilon}{56}} Y^{\frac{13}{14}} \geqslant X^{1+\varepsilon} Y^{\frac{13}{14}} .
\end{gathered}
$$

This finally completes the proof of Theorem 2.1.

\section{Proof of Theorems 2.2 And 1.1}

Proof of Theorems 2.2. The idea is to apply Theorems 2.1 in a delicate way. Trivially we have $r_{4}^{*}(d) \leqslant d \tau(d)$ (here $\tau(n)$ is the divisor function), and therefore

$$
S(x, y) \leqslant y \sum_{n \leqslant x} \sum_{d \mid n^{4}} \tau(d) \ll x y(\log x)^{14}
$$

for all $x \geqslant 2$ and $y \geqslant 2$, where the implied constant is absolute.

Let $\delta:=1-(\log B)^{-1}$ and let $k_{0}$ be a positive integer such that $\delta^{k_{0}}<(\log B)^{-3} \leqslant$ $\delta^{k_{0}-1}$. Note that $k_{0} \asymp(\log B) \log \log B$. In view of $(5.1)$, we can write

$$
\begin{aligned}
T(B) & =\sum_{1 \leqslant k \leqslant k_{0}} \sum_{\delta^{k}} \sum_{B<n \leqslant \delta^{k-1} B} \sum_{\substack{d \mid n^{4} \\
d<n^{4} / B^{2}}} r_{4}^{*}(d) \mathbb{1}_{\square}\left(\frac{n^{4}}{d}\right)+O\left(B^{3}\right) \\
& \leqslant \sum_{1 \leqslant k \leqslant k_{0}}\left(S\left(\delta^{k-1} B, \delta^{4(k-1)} B^{2}\right)-S\left(\delta^{k} B, \delta^{4(k-1)} B^{2}\right)\right)+O\left(B^{3}\right) .
\end{aligned}
$$

Similarly (even easily),

$$
T(B) \geqslant \sum_{1 \leqslant k \leqslant k_{0}}\left(S\left(\delta^{k-1} B, \delta^{4 k} B^{2}\right)-S\left(\delta^{k} B, \delta^{4 k} B^{2}\right)\right) .
$$

By (2.5) of Theorem 2.1 with $\eta=\frac{1}{4}$, a simple computation shows that

$$
\begin{aligned}
& T(B) \leqslant 2(1-\delta) \frac{1-\delta^{5 k_{0}}}{1-\delta^{5}} \cdot \mathscr{C}_{4} B^{3} \log B\left\{1+O\left(\frac{1}{\sqrt[4]{\log B}}\right)\right\}+O\left(B^{3}\right), \\
& T(B) \geqslant 2\left(\delta^{-1}-1\right) \frac{\delta^{5}-\delta^{5\left(k_{0}+1\right)}}{1-\delta^{5}} \cdot \mathscr{C}_{4} B^{3} \log B\left\{1+O\left(\frac{1}{\sqrt[4]{\log B}}\right)\right\} .
\end{aligned}
$$


By noticing that

$$
\begin{gathered}
(1-\delta) \frac{1-\delta^{5 k_{0}}}{1-\delta^{5}}=\frac{1-\delta^{5 k_{0}}}{1+\delta+\delta^{2}+\delta^{3}}=\frac{1}{5}+O\left(\frac{1}{\sqrt[4]{\log B}}\right) \\
\left(\delta^{-1}-1\right) \frac{\delta^{5}-\delta^{5\left(k_{0}+1\right)}}{1-\delta^{5}}=\frac{\delta^{4}-\delta^{5 k_{0}+4}}{1+\delta+\delta^{2}+\delta^{3}+\delta^{4}}=\frac{1}{5}+O\left(\frac{1}{\sqrt[4]{\log B}}\right) .
\end{gathered}
$$

The desired asymptotic formula (2.7) follows from (5.4) and (5.5).

Proof of Theorem 1.1. Applying (2.4) of Theorem 2.1 with $(x, y)=\left(B, B^{2}\right)$, we have

$$
\sum_{n \leqslant B} \sum_{\substack{d \mid n^{4} \\ d \leqslant B^{2}}} r_{4}^{*}(d) \mathbb{1}_{\square}\left(\frac{n^{4}}{d}\right)=2 \mathscr{C}_{4} B^{3} \log B\left\{1+O\left(\frac{1}{\sqrt[4]{\log B}}\right)\right\} .
$$

Inserting this and (2.7) into (2.2), we obtain (1.5) with $\mathcal{C}_{4}^{*}=\frac{192}{5} \mathscr{C}_{4}$.

Finally (1.4) follows from (1.5) via the inversion formula of Möbius.

Acknowledgements. This work was supported by National Natural Science Foundation of China (Grant Nos. 11531008 and 11771121) and the Program PRC 1457AuForDiP (CNRS-NSFC).

\section{REFERENCES}

[1] V. Batyrev and Y. Tschinkel, Tamagawa numbers of polarized algebraic varieties, Astérisque 251 (1998), 299-340.

[2] R. de la Bretèche, Sur le nombre de points de hauteur bornée d'une certaine surface cubique singulière, Astérisque 251 (1998), 51-77.

[3] R. de la Bret èche, T. Browning and P. Salberger, Counting rational points on the Cayley ruled cubic, European Journal of Mathematics 2 (2016), 55-72.

[4] R. de la Bretèche, J. Liu, J. Wu and Y. Zhao. On a certain non-split cubic surface, arXiv: 1709.09476.

[5] E. Grosswald, Representations of integers as sums of squares, Springer-Verlag, New York, 1985. xi+251 pp. ISBN: 0-387-96126-7.

[6] H. Iwaniec, Topics in classical automorphic forms, Graduate Studies in Mathematics, vol. 17, American Mathematical Society, Providence, Rhode Island, 1997.

[7] J. Liu, J. Wu and Y. Zhao, Manin's conjecture for a class of singular cubic hypersurfaces. IMRN, Vol. 2017, No. 00, pp 1-36, doi: 10.1093/imrn/rnx179.

[8] G. Tenenbaum, Introduction to analytic and probabilistic number theory, Translated from the second French edition (1995) by C. B. Thomas, Cambridge Studies in Advanced Mathematics 46, Cambridge University Press, Cambridge, 1995. xvi+448 pp.

Jianya Liu, School of Mathematics, Shandong University, Jinan, Shandong 250100, CHInA

E-mail address: jyliu@sdu.edu.cn

Jie Wu, CNRS UMR 8050, LAboratoire D'ANAlyse et DE MAThÉmatiques appliquÉEs, Université Paris-Est Créteil, 94010 Créteil Cedex, France

E-mail address: jie.wu@math.cnrs.fr

Yongqiang Zhao, Westlake University, School of Science, Shilongshan Road, Cloud Town, Xihu District, Hangzhou, Zhejiang 310024, China

E-mail address: yzhao@wias.org.cn 